\title{
Aplicabilidade científica do método dos elementos finitos
}

\author{
Raquel S. Lotti* , André Wilson Machado**, Ênio Tonani Mazzieiro***, Janes Landre Júnior ${ }^{\star \star \star \star ~}$
}

\begin{abstract}
Resumo
O Método dos Elementos Finitos (MEF) é uma análise matemática que consiste na discretização de um meio contínuo em pequenos elementos, mantendo as mesmas propriedades do meio original. Esses elementos são descritos por equações diferenciais e resolvidos por modelos matemáticos, para que sejam obtidos os resultados desejados. A origem do desenvolvimento deste recurso ocorreu no final do século XVIII, entretanto, a sua viabilização tornouse possível somente com o advento dos computadores, facilitando a resolução das enormes equações algébricas. O MEF pode ser utilizado em diversas áreas das ciências exatas e biológicas e, devido à sua grande aplicabilidade e eficiência, existem trabalhos com esta metodologia nas diversas especialidades odontológicas, como na Ortodontia, quando se deseja analisar cargas, tensões ou deslocamentos. Com o contínuo uso desse método em pesquisas, com suas vantagens em relação a outros disponíveis, torna-se de suma importância o conhecimento da técnica, para que sua utilização possa proporcionar benefícios científicos à Ortodontia. Tornase primordial que os ortodontistas clínicos conheçam os conceitos básicos do MEF para que os resultados dos trabalhos sejam melhor interpretados.
\end{abstract}

Palavras-chave: Método dos Elementos Finitos. Ortodontia. Pesquisa científica.

\section{INTRODUÇÃO}

O desenvolvimento do Método dos Elementos Finitos (MEF) teve suas origens no final do século XVIII, quando Gauss propôs a utilização de funções de aproximação para a solução de problemas matemáticos ${ }^{11}$. Durante mais de um século, diversos matemáticos desenvolveram teorias e técnicas analíticas para a solução de problemas, entretanto, pouco se evoluiu devido à dificuldade e à limitação existente no processamento de equações algé- bricas $^{6}$. O desenvolvimento prático desta análise ocorreu somente muito mais tarde em conseqüência dos avanços tecnológicos, por volta de 1950, com o advento da computação. Isto permitiu a elaboração e a resolução de sistemas de equações complexas ${ }^{6}$. Em 1956, Turner, Clough, Martins e Topp, trabalhando em um projeto de aeronaves para a Boeing, propuseram um método de análise estrutural, similar ao MEF. Mais tarde, em 1960, estes autores utilizaram pela primeira vez o nome

* Mestre em Ortodontia pela PUC-Minas.

** Mestrando em Ortodontia pela PUC-Minas.

*** Doutor em Ortodontia, Coordenador do curso de Mestrado em Ortodontia da PUC-Minas.

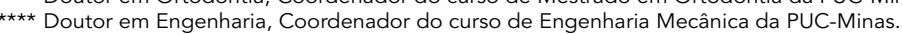


de Método dos Elementos Finitos, descrevendo-o. A partir de então, seu desenvolvimento foi exponencial, sendo aplicado em diversas áreas da Engenharia, Medicina, Odontologia e áreas afins ${ }^{11}$.

Em linhas gerais, pode-se definir o MEF como um método matemático, no qual um meio contínuo é discretizado (subdividido) em elementos que mantém as propriedades de quem os originou. Esses elementos são descritos por equações diferenciais e resolvidos por modelos matemáticos para que sejam obtidos os resultados desejados. O MEF é utilizado há algum tempo em experimentos relacionados à Odontologia, em diversas especialidades, sendo a sua aplicação na Ortodontia de grande utilidade $8,9,10,12,14,15,16,18,19,20,21,22,23,24,25$.

O estudo do efeito das cargas (forças) aplicadas aos dentes apresenta grande interesse científico e pode ser encontrado em diversos trabalhos, envolvendo metodologias variadas $2,3,5,7,9,14$. Dentre as principais metodologias utilizadas, pode-se destacar: métodos convencionais para a análise de tensões na estrutura dentária, como modelos fotoelásticos e estudos com laser holográficos; modelos matemáticos analíticos; análises experimentais em humanos e/ou animais e análises matemáticas como o MEF.

Os métodos convencionais podem ser questionados principalmente devido à incapacidade de criar modelos semelhantes à estrutura dentária, devido à diversidade de substâncias que compõem os dentes e à irregularidade de seu contorno. Esses estudos necessitam de laboratórios bem equipados e instrumentos específicos, dificultando a realização do experimento e aumentando seu custo $^{2,3}$. Modelos fotoelásticos seriam ainda limitados pela simplificação das suposições, uma vez que, consideram muitas vezes apenas um plano bidimensional, representam formas geométricas ideais e não reais, e não consideram a mudança de direção da força durante o deslocamento do dente ${ }^{2}$. Além disso, Rubin et al. ${ }^{15}$ relataram também que métodos fotoelásticos são complexos e resultados numéricos poderiam ser mais facilmente obtidos por outros meios, como o MEF.

Os métodos envolvendo laser holográfico consideram as propriedades não lineares do ligamento periodontal, entretanto, estas serão diferentes do tecido in vivo, devido à forma como a estrutura é simulada nesta técnica, não sendo possivel criar materiais exatamente com as mesmas respostas desta estrutura. Além disso, nesta técnica, não é possível variar as formas geométricas utilizadas ${ }^{2}$.

As análises matemáticas analíticas podem representar situações in vivo, expressando, mediante equações matemáticas compatíveis com o real, a forma, o contorno e a função do modelo ${ }^{17}$. Entretanto, os resultados oferecidos por estes modelos são, de certa forma, simplificados, desconsiderando sempre algum fator, como por exemplo, a largura ou a área do dente, que pode possuir maior ou menor influência, dependendo do tipo de análise.

As técnicas experimentais em humanos e/ou animais também são limitadas devido à variabilidade de fatores que podem conduzir a erros quando se aplicam complexos sistemas de forças em seres vivos, como por exemplo: 1) inabilidade de calcular precisamente a distribuição da tensão e compressão no ligamento periodontal; 2) ocorrência de falhas durante o controle do tipo de movimento dentário; 3) dificuldade de avaliação de todas as fases do movimento; 4) presença de grandes variações individuais dificultando a análise dos resultados ${ }^{13}$.

Uma possível solução para a realização destes estudos seria a utilização do MEF. Este método de pesquisa possui a capacidade de modelar matematicamente estruturas complexas com geometrias irregulares de tecidos naturais e artificiais, como os dentes e os diversos biomateriais usados em Ortodontia, bem como modificar os parâmetros de sua geometria. Com isso, torna-se possível a aplicação de um sistema de forças em qualquer ponto e/ou direção, promovendo, assim, informações sobre o deslocamento e o grau de tensão provocado por essas cargas ao elemento dentário ou o tecido analisado ${ }^{11,13,22,25}$. 
Devido ao contínuo uso desse método em pesquisas e às vantagens em relação a outros disponíveis, torna-se de suma importância o conhecimento da técnica, para que sua utilização possa proporcionar benefícios às pesquisas científicas em Ortodontia. Além disso, torna-se primordial que os ortodontistas clínicos conheçam os conceitos básicos do MEF para que os resultados das crescentes pesquisas possam ser mais bem entendidos, interpretados e empregados no diagnóstico, planejamento e tratamento das más oclusões.

$\mathrm{O}$ intuito desse trabalho foi discorrer sobre o Método dos Elementos Finitos, caracterizando todas as fases necessárias para a obtenção do modelo experimental e a correta interpretação dos resultados.

\section{A OBTENÇÃO DO MODELO EXPERIMENTAL}

Para a obtenção de um modelo experimental por meio do MEF, é necessário definir o objeto de pesquisa, que poderá ser qualquer estrutura dento-maxilo-facial. No primeiro passo, define-se a geometria da estrutura que se deseja analisar, como por exemplo um canino e seu suporte alveolar (Fig. 1A, B). O objeto é desenhado graficamente em um programa de computador específico, como por exemplo o SolidWorks (SolidWorks Corporation, EUA) ou AutoCAD (Autodesk, EUA) (Fig. 1A, B). A morfologia das estruturas modeladas pode ser baseada em Atlas de Anatomia, tomografias computadorizadas, crânios secos e/ou dentes extraídos.

Posteriormente, a estrutura criada será discretizada em pequenos elementos denominados elementos finitos em um programa específico de MEF, como por exemplo, o Patran e Nastran (MSC Software, EUA) ou o Cosmos (SolidWorks Corporation, EUA). Os elementos representam coordenadas no espaço e podem assumir diversos formatos, sendo que os tetraédricos e os hexaédricos são os mais comuns (Fig. 2) ${ }^{6}$. Quanto maior o número de elementos mais preciso será o modelo ${ }^{10}$.

Nas extremidades de cada elemento finito encontram-se pontos, ou nós, que conectam os elementos entre si, formando uma malha arranjada em camadas bi ou tridimensionais (Fig. 2). Através dos nós as informações são passadas entre os elementos. Cada nó possui um número definido de graus de liberdade, que caracterizam a forma como o nó irá deslocar-se no espaço. Este deslocamento pode ser descrito em três dimensões espaciais (X, $\mathrm{Y}$ e Z) no caso de modelos tridimensionais, ou duas direções ( $\mathrm{X}$ e Y) em modelos bidimensionais. A direção e o sentido das coordenadas são determinados pelo pesquisador. Portanto, pode-se definir, por exemplo, o eixo $\mathrm{X}$ correspondente às alterações no plano coronal (ântero-posteriores), o Y referente às mudanças no plano sagital mediano (transversais) e o $\mathrm{Z}$ no plano axial (verticais). Estes serão os eixos utilizados para a avaliação dos resultados.

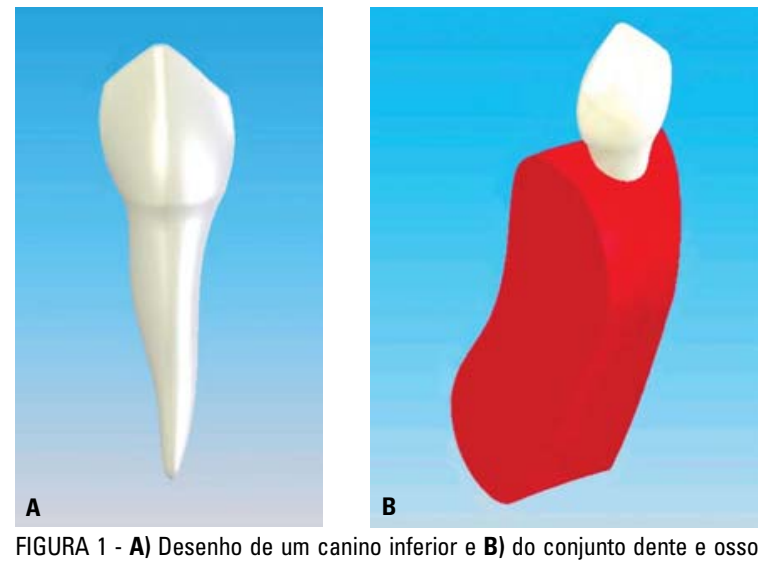
alveolar, modelados pelo software SolidWorks (SolidWorks Corporation, EUA).

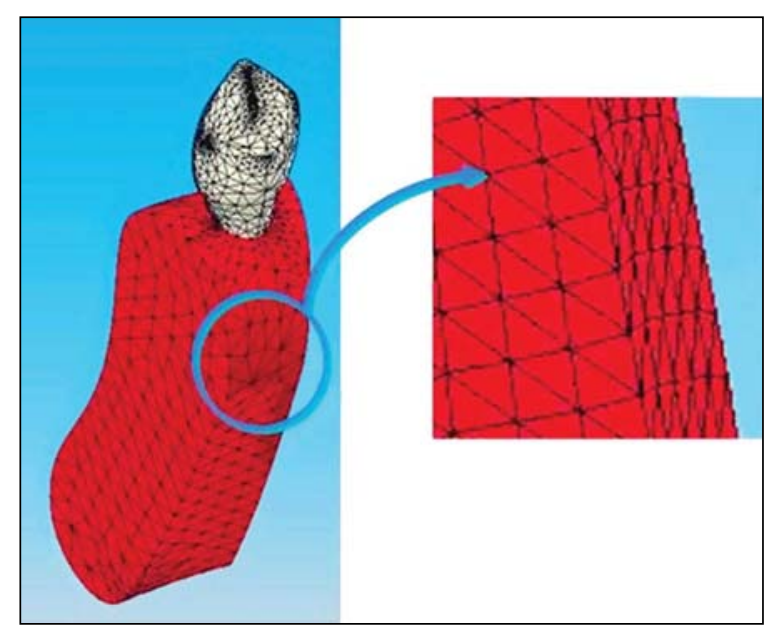

FIGURA 2 - Malha do canino e osso alveolar desenvolvidas. Nessa ilustração cada triângulo representa um elemento finito, inter-conectados pelos nós localizados nas extremidades. 
O arranjo dos nós possui graus de liberdade que o caracterizam como tridimensionais ou bidimensionais. Modelos tridimensionais possuem vantagens sobre os bidimensionais, uma vez que somente no primeiro é possivel analisar corretamente as estruturas dento-maxilo-faciais. Por exemplo, a irregularidade dos dentes humanos, as cargas aplicadas sobre estes, a distribuição das tensões e deslocamentos sobre as várias estruturas que compõem o dente, como esmalte, dentina e polpa, e os resultados nos três planos do espaço $^{15}$.

\section{PROPRIEDADES DO MODELO EXPERIMENTAL}

O próximo passo é a determinação das propriedades físicas e mecânicas de cada estrutura constituinte do modelo. Esta etapa é muito importante para se obter fidelidade dos resultados, uma vez que as características de cada componente do modelo influenciarão o comportamento das respostas às aplicações das forças.

Característica de fundamental importância é o comportamento dos materiais frente a uma deformação. Nessas situações, diversos fenômenos podem ocorrer: fenômenos elásticos não-lineares (após a deformação ocorre retorno à condição de origem sem seguir um padrão), fenômenos plásticos (a deformação ocorre sem retorno à condição de origem), fenômenos elasto-plásticos (parte da observação possui comportamento elástico e parte plástico), fenômenos viscoelásticos (ocorre deformação e o retorno à origem é considerado dependente do tempo) e fenômenos viscoplásticos (ocorre deformação sem retorno à condição de origem e é dependente do tempo).

O movimento dentário se encaixa dentro dos fenômenos viscoplásticos, ou seja, o movimento do dente é dependente do tempo, e após a remoção da força ele não retorna completamente à sua posição de origem. O trabalho de Tanne Inoue e Sakuda ${ }^{19}$ demonstrou que alterações do comportamento biomecânico do ligamento periodontal e do osso alveolar estão presentes após o movimento dentá- rio, levando a uma diminuição da natureza elástica destas estruturas. Desta forma, estudos incluindo as propriedades viscoplásticas e viscoelásticas que consideram o fator tempo seriam ideais (principalmente as do ligamento periodontal), entretanto não se conhecem plenamente estas características, limitando sua aplicação ${ }^{12}$. Para a realização de uma pesquisa com estas características, seria necessária a dedução da maneira como as estruturas se comportam, sem poder comprovar a veracidade dos resultados. Outra opção mais adequada seria realizar estudos sistemáticos e precisos in vivo para determinar estas propriedades com precisão e validade, entretanto, os métodos existentes são invasivos. Por estes motivos, a maioria dos trabalhos utiliza apenas modelos linearmente elásticos em suas análises. Nesse sistema, as deformações da estrutura são diretamente proporcionais às forças aplicadas.

A utilização destes tipos de modelos permite apenas a avaliação do deslocamento inicial dos dentes (antes de ocorrer os fenômenos celulares levando à remodelação óssea), ou seja, sua tendência de movimento, desconsiderando o fator tempo.

Além destas propriedades de elasticidade, os materiais podem ser considerados como isotrópicos, anisotrópicos ou ortotrópicos. Um material isotrópico significa que suas propriedades mecânicas são as mesmas em todas as direções em um mesmo ponto do elemento estrutural. Em um material ortotrópico, suas propriedades mecânicas são as mesmas em duas direções e diferentes em uma terceira, enquanto em um material anisotrópico, as propriedades diferem em todas as direções.

Por fim, determina-se o Coeficiente de Poisson e o Módulo de Young (Elasticidade) das estruturas. O Coeficiente de Poisson refere-se ao valor absoluto da relação entre as deformações transversais e longitudinais em um eixo de tração axial, e o Módulo de Young representa a inclinação da porção linear do diagrama de tensão/deformação do material. A tabela 1 ilustra os valores destas propriedades nas principais estruturas dentárias. 
Tabela 1 - Propriedades mecânicas dos materiais.

\begin{tabular}{|c|c|c|}
\hline Material & $\begin{array}{l}\text { Módulo de } \\
\text { Young }\end{array}$ & $\begin{array}{l}\text { Coeficiente de } \\
\text { Poisson }\end{array}$ \\
\hline Esmalte $^{15}$ & $4,1 \times 10^{4} \mathrm{MPa}$ & 0,30 \\
\hline Dentina $^{15}$ & $1,9 \times 10^{4} \mathrm{MPa}$ & 0,31 \\
\hline Polpa $^{15}$ & $2,07 \mathrm{MPa}$ & 0,45 \\
\hline Osso cortical ${ }^{11}$ & $1,37 \times 10^{4} \mathrm{MPa}$ & 0,30 \\
\hline Osso esponjoso ${ }^{11}$ & $1,37 \times 10^{3} \mathrm{MPa}$ & 0,30 \\
\hline Ligamento periodontal' & $7 \times 10^{-2} \mathrm{MPa}$ & 0,49 \\
\hline
\end{tabular}

Dependendo do objetivo da pesquisa, nem sempre é necessário separar todas estas estruturas em um modelo experimental. De acordo com Rubin et al. ${ }^{15}$, o impacto do ligamento periodontal perante a distribuição das tensões, no MEF, é pequeno, fazendo com que esta estrutura possa ser desprezada durante a modelagem. Entretanto, quando se objetiva analisar as tensões geradas nesta membrana, sua modelagem deverá ser realizada. Estes mesmos autores relatam ainda que a polpa apresenta um suporte muito pequeno à carga e os valores de suas propriedades poderiam ser desconsiderados quando comparados aos dos outros tecidos. Portanto, sua modelagem não resultaria em diferenças significantes no resultado, não justificando sua inclusão na maioria dos casos. A divisão entre osso cortical e esponjoso também já foi discutida na literatura. Segundo Andersen, Pedersen e Melsen ${ }^{1}$, as alterações dos resultados, considerando ou não esta divisão, são muito pequenas, permitindo a simplificação do modelo em apenas um tipo de osso alveolar.

Quanto mais estruturas forem modeladas, mais precisos serão os resultados, porém a obtenção do modelo será mais difícil e a análise dos resultados mais complexa. Entretanto, baseado no tipo de análise a ser elaborada, pode-se utilizar modelos mais simplificados e obter os mesmos resultados qualitativos. Portanto, esta modelagem deverá ser avaliada criteriosamente, a fim de simplificar o modelo, de acordo com suas reais necessidades e sem comprometer os resultados.

\section{ANÁLISE DOS RESULTADOS}

Após a determinação de todas as propriedades, realiza-se a aplicação das cargas necessárias e a análise dos resultados. O comportamento de cada um dos elementos é descrito por funções algébricas, em que os achados representarão a distribuição das tensões e deformações do modelo. Os principais softwares utilizados para esta etapa são o Patran e Nastran e o Cosmos.

A visualização dos resultados é feita por uma escala de cores, em que cada tonalidade, corresponde a uma quantidade de deslocamento ou tensão gerada nas estruturas (Fig. 3, 4, 5). Desta forma, pode-se detectar como ocorreu o deslocamento do dente (ou de qualquer estrutura estudada), o tipo de movimento realizado por este, qual região se deslocou em maior magnitude, ou como as tensões se distribuiriam sobre as estruturas analisadas (dentes, osso alveolar, ligamento periodontal ou qualquer outro objeto de análise) nas três direções do espaço (X, Y e Z), na presença de um modelo tridimensional, ou somente em duas direções ( $\mathrm{X}$ e Y) em modelos bidimensionais. Quando selecionado um dos eixos da coordenada (X, Y ou Z), a escala de cores e seus valores corresponderão à quantidade de tensão e/ou deslocamento presente na direção e no sentido do eixo avaliado, conforme previamente definido.

Ainda com este método, é possível obter o deslocamento em magnitude, ou seja, o maior deslocamento de cada ponto em direção à resultante dos deslocamentos existentes, sendo independente de seu sentido (Fig. 4). Outro tipo de análise das tensões é chamado de Von Mises, que representam a média das tensões em todas as direções. Os resultados nessa situação permitem a localização dos pontos de maior tensão (Fig. 5).

De acordo com Cook, Malkus e Plesha ${ }^{4}$, as etapas a serem seguidas para a obtenção dos resultados são as seguintes: 1) divisão da estrutura em elementos finitos com a ajuda de programas pré-processadores; 2) formulação das propriedades de cada elemento; 3) reunião dos elementos para a obtenção de um 


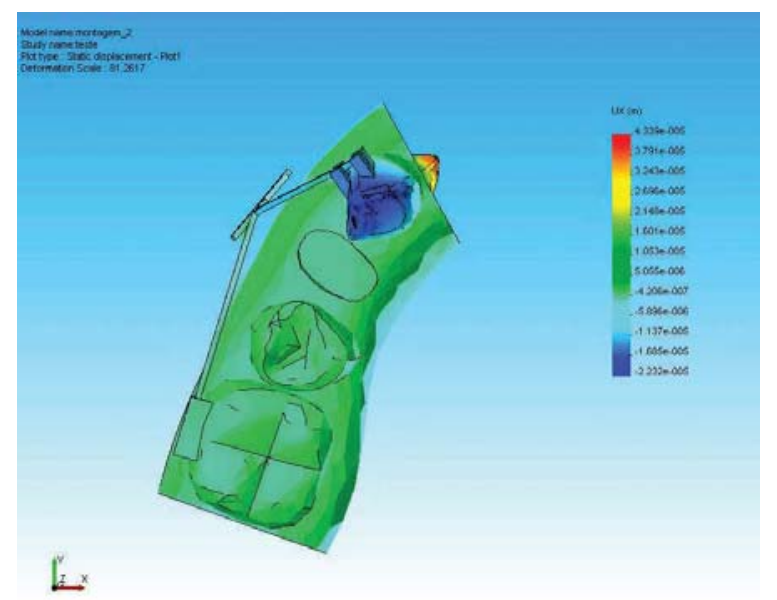

FIGURA 3 - Deslocamento do canino inferior esquerdo na direção " $x$ " durante a aplicação do efeito mola do aparelho. São apresentados valores negativos e positivos de acordo com o sentido do deslocamento.

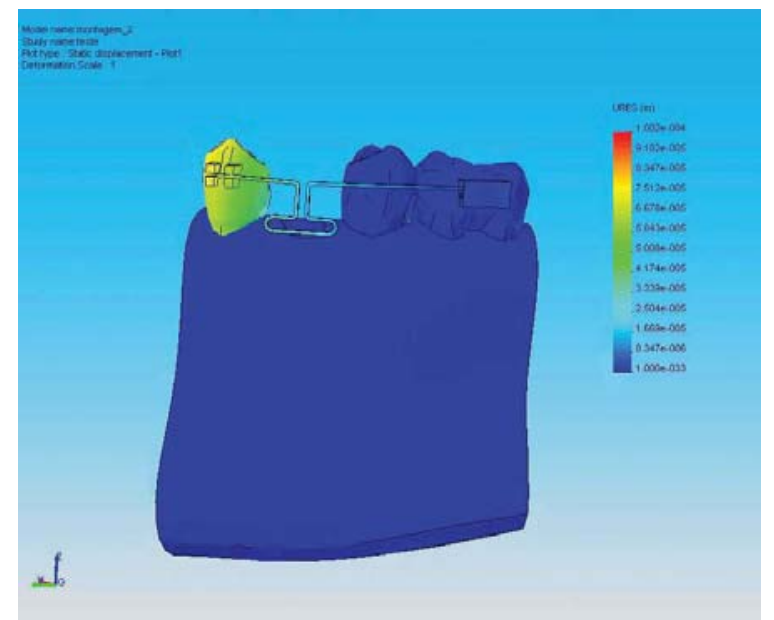

FIGURA 4 - Análise do deslocamento dentário em magnitude durante a avaliação de determinada mecânica.

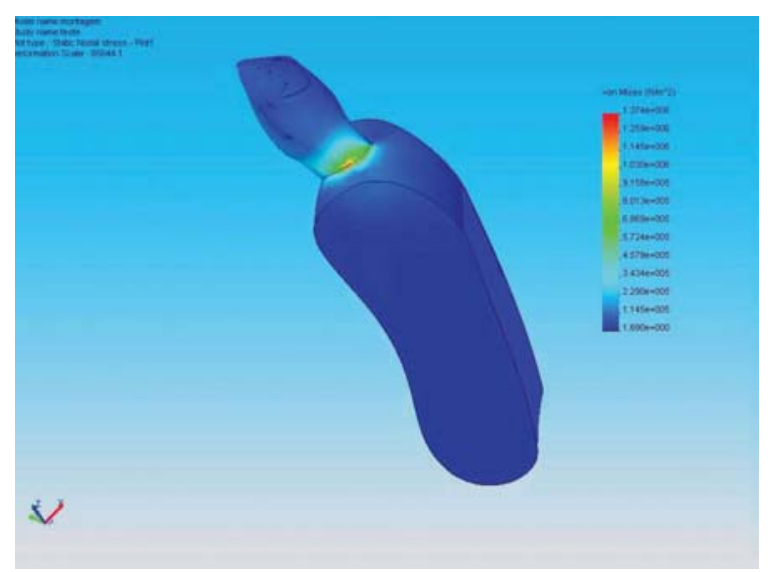

FIGURA 5 - Análise da distribuição da tensão em Von Mises ao redor das estruturas envolvidas, após a aplicação de uma força simples. modelo de elementos finitos da estrutura; 4) aplicação das cargas necessárias; 5) especificação de como a estrutura se comporta; 6) resolução simultânea das equações algébricas e lineares; 7) cálculo das tensões e do deslocamento entre os elementos e interpretação, utilizando programas pós-processadores.

Por meio do MEF qualquer material ou estrutura dento-maxilo-facial pode ser modelada e os esforços analisados. Uma de suas principais vantagens consiste no fato de poder controlar qualquer variável relacionada ao experimento, facilitando a análise dos resultados, proporcionando grandes benefícios às pesquisas científicas.

Entretanto, apesar de todas as vantagens deste método, deve-se ter em mente que a precisão de seus resultados também possui limites de tolerância, que devem ser levados em consideração, como em todo modelo matemático. Oliveira ${ }^{11}$ comenta que os fatores que podem conduzir a imprecisões dos resultados são: 1) a variabilidade inerente aos processos biomecânicos, como por exemplo variações de tamanho ou forma do objeto de pesquisa ou de características mecânicas dos materiais; 2) simplificações para a adoção de um determinado modelo matemático; 3) a divisão de estruturas complexas em várias formas geométricas, levando à perda de alguns detalhes. Cook, Malkus e Plesha $^{4}$, ainda ressaltam que outras situações também poderiam levar a erros durante a execução do programa, como: 1) omissão ou má interpretação de aspectos importantes do comportamento físico do material; 2) erros do programa de computador não checados devidamente; 3) utilização de programas inapropriados e/ou de informações incorretas; 4) obtenção de uma malha muito simplificada; 5) uso de um elemento inadequado.

\section{APLICAÇÃO DO MÉTODO DOS ELEMEN- TOS FINITOS EM ORTODONTIA}

Diversos são os trabalhos encontrados na literatura utilizando o MEF. Entre suas variadas aplicações pode-se citar alguns exemplos como: o estudo da distribuição das tensões durante o movimento den- 
tário $^{18}$, o efeito de forças ortopédicas no complexo craniofacial $^{20}$, superposições cefalométricas ${ }^{16}$, a verificação das cargas exercidas em implantes como recurso de ancoragem ${ }^{23}$, a avaliação da resistência de base de braquetes ${ }^{25}$ e a avaliação do desempenho de molas para fechamento de espaços ${ }^{8}$.

Tanne e Sakuda ${ }^{21}$ utilizaram técnicas histológicas e matemáticas (MEF) em um mesmo objeto de estudo, com o intuito de comparar os resultados, e encontraram similaridade entre as metodologias aplicadas. Além disso, os autores afirmaram que os resultados de uma análise tridimensional, quando comparada com uma bidimensional, foram melhor elucidados. Um modelo tridimensional permite uma avaliação dos três planos do espaço e, como os movimentos ortodônticos são muito complexos, uma avaliação mais precisa dos resultados pode ser obtida.

Um exemplo interessante das aplicações do MEF pode ser constatado no trabalho de Tanne, Burstone e Sakuda ${ }^{18}$. Os autores modelaram um incisivo central, seu respectivo ligamento periodontal e osso alveolar para o estudo da distribuição das tensões nestas estruturas, após a aplicação de uma força. Por meio deste método foi possivel variar a altura do osso alveolar, simulando diferentes níveis de suporte periodontal, e comparar os resultados com diferentes comprimentos de raiz do mesmo elemento dentário, demonstrando a versatilidade do MEF em pesquisas científicas. Caso o mesmo estudo fosse realizado por outro método, como por exemplo laser holográfico, seriam necessários uma aparelhagem e suporte muito mais complexo, dificultando a execução do mesmo.

Middleton, Jones e Wilson ${ }^{10}$ investigaram por meio do MEF, utilizando um modelo em 3-D (três dimensões), as respostas biomecânicas de um canino superior submetido à aplicação de forças em sua coroa. A investigação demonstrou que informações quantitativas em relação ao deslocamento inicial deste elemento dentário, além da distribuição das tensões ao seu redor, podem ser previstas de forma precisa e utilizadas para avaliar as respostas do tra- tamento ortodôntico. Os autores justificaram a hipótese de um comportamento linearmente elástico dos materiais, ou seja, o material deforma-se seguindo um padrão e é capaz de voltar à sua condição de origem. Portanto, somente o deslocamento inicial é avaliado, uma vez que o fator tempo não tem como ser considerado em modelos linearmente elásticos.

Mc Guinness et al. ${ }^{9}$ realizaram um estudo por meio do MEF avaliando as tensões geradas no ligamento periodontal por aparelhos tipo edgewise. Os autores relataram que resultados reais são difíceis de serem determinados experimentalmente com o mesmo grau de exatidão in vivo, porém a precisão numérica obtida com o MEF pode ser repetida e comparada com outros estudos, justificando sua aplicabilidade e eficiência em pesquisas científicas. As vantagens deste método em relação a outros, como por exemplo modelos fotoelásticos, seriam inúmeras, dentre estas a relativa facilidade de se modelar estruturas geométricas complexas e irregulares de tecidos naturais e biomateriais de diferentes propriedades. Além disso, o programa permite a simulação de diversas magnitudes de força em diferentes pontos de aplicação.

Tanne, Matsubara e Sakuda ${ }^{20}$ utilizaram o MEF para avaliar a resposta das suturas da face diante da aplicação de uma força ortopédica por um aparelho extrabucal cervical. Os autores modelaram o complexo craniofacial de um crânio seco, aplicaram as cargas necessárias e demonstraram, por meio deste método, o deslocamento do complexo maxilar e a distribuição das tensões sobre as suturas da face. Desta forma, pôde-se obter informações mais precisas sobre o comportamento dos tecidos analisados, contribuindo para a otimização do uso deste aparelho.

Sameshima e Melnick ${ }^{16}$ realizaram superposições cefalométricas durante o tratamento e após seu término. Relataram que, com o MEF, as alterações em tamanho, forma e posição das estruturas da face são facilmente visualizadas, ajudando na interpretação dos resultados.

Um estudo realizado por Provatidis ${ }^{12}$ analisou 
a resposta do movimento dentário (localização dos centros de resistência e rotação) após a aplicação de cargas, utilizando diferentes propriedades do ligamento periodontal, sendo consideradas cinco diferentes propriedades deste tecido. O primeiro modelo foi composto por um ligamento periodontal isotrópico e linearmente elástico, desconsiderando suas fibras, outros três modelos não lineares e anisotrópicos compostos de fibras periodontais arranjadas em três diferentes orientações, e um quinto modelo considerando o ligamento como ortotrópico, composto de fibras e estrutura contínua. Baseado nos resultados, o autor pôde comparar e concluir sobre a melhor maneira de se modelar esta estrutura em trabalhos científicos e afirmou que todos os tipos de ligamento se encontravam dentro de uma margem de erro clinicamente aceitável.

Vásquez et al. ${ }^{23}$ modelaram um implante na região posterior, e um canino superior, para avaliar as cargas impostas a estas estruturas e no ligamento periodontal do elemento dentário. Para tal, foram simulados dois sistemas de forças para a retração deste elemento dentário, o primeiro semelhante à mecânica de deslizamento e o último similar à técnica segmentada. Aplicaram forças simples em ambas as estruturas (implante e canino) para simular a mecânica de deslizamento e sistemas de forças mais complexos (força e momento) para simular uma alça "T" segmentada. Compararam os resultados e verificaram os diferentes comportamentos das estruturas nas diferentes mecânicas empregadas.

Viana ${ }^{24}$ avaliou, por meio do MEF, a resistência ao deslocamento de quatro diferentes bases de braquetes ortodônticos, de diferentes marcas comerciais, durante a aplicação de forças de torção e cisalhamento. Com essa metodologia, foi possivel obter respostas quanto ao comportamento das diferentes bases de braquetes, ao tipo de interface (braquete/adesivo ou adesivo/esmalte) mais sujeita à falha e ao tipo de força mais provável de causar insucesso na união.

Lotti $^{8}$ investigou o comportamento da retração de caninos inferiores por meio da técnica segmentada em um modelo tridimensional. Foi modelado o hemi-arco inferior esquerdo com o canino, segundo pré-molar, primeiro molar, espaço referente à "extração" do primeiro pré-molar e três alças "T" segmentadas ligando o canino ao molar. As disposições dessas alças diferiam e apresentavam-se em três situações: próxima ao canino, centralizada e próxima ao molar. Por meio do MEF, obtiveram-se respostas em relação à quantidade de deslocamento destes elementos dentários, bem como, magnitude de rotação, inclinação e extrusão.

\section{CONSIDERAÇÕES FINAIS}

Por meio do MEF, inúmeros trabalhos com diferentes aplicações e objetivos podem ser conduzidos, sendo o mesmo plenamente aplicável para a realização de pesquisas científicas em Ortodontia. Este método, quando bem gerenciado, pode proporcionar diversas vantagens em relação a outros estudos, pela facilidade de obtenção e interpretação dos resultados. Entretanto, para a correta execução desta metodologia, é necessária a interação entre profissionais da Engenharia e da Odontologia para que se possa por em prática as idéias e obter resultados corretos e válidos. 


\title{
Scientific aplication of finite element method
}

\begin{abstract}
The Finite Element Method consists of a series of mathematic equations that, utilized with the proper software, allows different kinds of computer simulations. The method was first reported in the end of the seventeenth century, however it's practical utilization was made feasible only after the advent of computers. Scientific researches that works with this analysis is granted with many advantages. Especially in the Orthodontics field this approach can be very useful to evaluate the forces delivered by different orthodontic appliances, tooth movement and structures of the face. Due to it's widespread use in orthodontic research it is of paramount importance to know well the method and its limitations to provide the proper background to the clinician and the researcher.
\end{abstract}

Key words: Finite Elements Analysis. Orthodontics. Scientific research.

\section{REFERÊNCIAS}

1. ANDERSEN, K. L.; PEDERSEN, E. H.; MELSEN, B. Material parameters and stress profiles within the periodontal ligament. Am J Orthod Dentofacial Orthop, St. Louis, v. 99, no. 5, p. 427-40, May, 1991

2. BURSTONE, C. J.; PRYPUTNIEWICZ, R. J. Holographic determination of centers of rotation produced by orthodontic forces. Am J Orthod, St. Louis, v. 77, no. 4, p. 398-409, Apr. 1980.

3. CAPUTO, A. A.; CHACONAS, S. J.; HAYASHI, R. K. Photoelastic visualization of orthodontic forces during canine retraction. Am J Orthod, St. Louis, v. 65, no. 3, p. 250-259, Nov. 1974.

4. COOK, R. D.; MALKUS, D.; PLESHA, M. E. Introduction. In: _. Concepts and aplications of finite element analysis. $3 . \mathrm{ed}$. John Willey \& Sons, 1989. cap. 1, p. 1-30.

5. FORTIN, J. M. Translation of premolars in the dog by controlling the moment-to force ratio on the crown. Am J Orthod, St. Louis, v. 59, no. 6, p. 541-551, Jun. 1971.

6. GALLAGHER $\mathrm{R}, \mathrm{H}$. Introduction In Finite element analysis: fundamentals. 4. ed. Englewood Cliffs: Prentice-Hall, 1975. cap. 1, p. 1-19.

7. LEE, B. W. Relationship between tooth-movement rate and estimated pressure applied. J Dent Res, Washington, v. 44, no. 5, p. 1053, Sept./Oct. 1965

8. LOTTI, R. S. A influência do posicionamento da alça segmentada em " $T$ " durante o movimento de retração inicial: uma avaliação pelo método dos elementos finitos. 2003. 137f. Dissertação (Mestrado em Ortodontia)-Faculdade de Odontologia, Pontifícia Universidade Católica de Minas Gerais, Belo Horizonte, 2003.

9. MC GUINNESS, N. et al. Stresses induced by edgewise appliances in the periodontal ligament: a finite element study. Angle Orthod, Appleton, v. 62, no. 1, p. 15-22, Jan. 1992.

10. MIDDLETON, J.; JONES, M. L.; WILSON, N. A. Three-dimensional analysis of orthodontic tooth movement. J Biomed Eng, Surrey, v. 12, no. 4, p. 319-327, Jul. 1990.

11. OLIVEIRA, E. J. Biomecânica básica para ortodontistas. 1. ed. Belo Horizonte: UFMG, 2000. 196p.

12. PROVATIDIS, C. A comparative FEM-study of tooth mobility using isotropic and anisotropic models of the periodontal ligament. Med Eng Phys, Oxford, v. 22, no. 5, p. 359-370, Jun. 2000.

13. REN, Y.; MALTHA, J. C.; KUIJPERS-JAGTMAN, A. M. Optimum force magnitude for orthodontic tooth movement: a systematic literature review. Angle Orthod, Appleton, v. 73, no. 1, p. 86-92, Jan. 2003.

14. REZENDE, I. C. B. Relação entre a perda de osso alveolar, magnitude de força aplicada e o deslocamento inicial do dente: uma análise tridimensional pelo método dos elementos finitos. 2000. 72 f. Monografia (Especialização em Ortodontia)Centro de Odontologia e Pesquisa da Pontifícia Universidade
Católica de Minas Gerais, Belo Horizonte, 2000.

15. RUBIN C. et al. Stress analysis of the human tooth using a threedimensional finite element model. J Dent Res, Washington, v. 62, no. 2, p. 82-86, Feb. 1983.

16. SAMESHIMA, G. T.; MELNICK, M. Finite element-based cephalometric analysis. Angle Orthod, Appleton, v. 64, no. 5, p. 343-350, 1994.

17. STEYN, C. L.; VERWOERD, W. S.; VAN DER MERWE, E. J.; FOURIE, O. L. Calculation of the position of the axis of rotation when single-rooted teeth are orthodontically tipped. $\mathbf{B r} \mathbf{J}$ Orthod Oxford, v. 5, no. 3, p. 153-156, Jul. 1978.

18. TANNE, K.; BURSTONE, C.; SAKUDA, M. Biomechanical responses of tooth associated with different root lengths and alveolar bone heights: changes of stress distributions in the PDL. J Osaka Univ Dent Sch, Osaka, v. 29, p. 17-24, Dec. 1989.

19. TANNE, K.; INOUE, Y.; SAKUDA, M. Biomechanical behavior of the periodontiun before and after orthodontic tooth movement. Angle Orthod, Appleton, v. 65, no. 2, p. 123-128, 1995

20. TANNE, L.; MATSUBARA, S.; SAKUDA, M. Stress distributions in the maxillary complex from orthopedic headgear forces. Angle Orthod, Appleton, v. 63, no. 2, p. 111-118, 1993.

21. TANNE, K.; SAKUDA, M. A dynamic analysis of stress in the tooth and its support structures: the use of the finite element method as numerical analysis (abstr.). J Japanese Orthod Sch Tokyo, v. 38, n. 4, p. 374-382, 1979.

22. TANNE, K.; SAKUDA, M.; BURSTONE, C. J. Three-dimensional finite element analysis for stress in the periodontal tissue by orthodontic forces. Am J Orthod Dentofacial Orthop, St. Louis, v. 92, n. 6, p. 499-505, Dec. 1987.

23. VÁSQUEZ, M. et al. Initial stress differences between sliding and sectional mechanics with an endosseous implant as anchorage: a 3-dimensional finite element analysis. Angle Orthod, Appleton, v. 71, n. 4, p. 247-56, Aug. 2001.

24. VIANA, C. P.; MAZZIEIRO, E. T.; LANDRE JR., J. A influência da variação da curvatura da base do braquete em uma união ortodôntica submetida a diferentes cargas, através do método dos elementos finitos. R Dental Press Ortodon Ortop Facial Maringá, v. 10, n. 3, p. 75-86, maio/junho 2005

25. WILLIAMS, K. R.; EDMUNDSON, J. T. Orthodontic tooth movement analyzed by the finite element method. Biomaterials, Guildford, v. 5, no. 6, p. 347-351, Nov. 1984

\section{Endereço para correspondência}

Raquel Silva Lotti

Av. das Américas, 3434, bloco 4, sala 515 - Barra da Tijuca

CEP: 30.535-610 - Rio de Janeiro/RJ

E-mail: raquelotti@ig.com.br 